FACTA UNIVERSITATIS

Series: Mechanical Engineering Vol. 14, N 3, 2016, pp. 269 - 280

DOI: 10.22190/FUME1603269T

Original scientific paper

\title{
ADHESION EFFECTS WITHIN THE HARD MATTER - SOFT MATTER INTERFACE: MOLECULAR DYNAMICS
}

UDC 539.8

\author{
Alexey Tsukanov ${ }^{1,2}$, Sergey Psakhie ${ }^{1,2}$ \\ ${ }^{1}$ Institute of Strength Physics and Materials Science, \\ Siberian Branch of Russian Academy of Sciences, Tomsk, Russian Federation \\ ${ }^{2}$ Tomsk Polytechnic University, Tomsk, Russian Federation
}

\begin{abstract}
In the present study three soft matter - hard matter systems consisting of different nanomaterials and organic molecules were studied using the steered molecular dynamics approach in order to reveal regularities in the formation of organic-inorganic hybrids and the stability of multimolecular complexes, as well as to analyze the energy aspects of adhesion between bio-molecules and layered ceramics. The combined process free energy estimation (COPFEE) procedure was used for quantitative and qualitative assessment of the considered heterogeneous systems. Interaction of anionic and cationic amino acids with the surface of a $\left[\mathrm{Mg}_{4} \mathrm{Al}_{2}(\mathrm{OH})_{12}{ }^{2+} 2 \mathrm{Cl}\right]$ layered double hydroxide ( $\mathrm{LDH}$ ) nanosheet was considered. In both cases, strong adhesion was observed despite the opposite signs of electric charge. The free energy of the aspartic amino acid anion, which has two deprotonated carboxylic groups, was determined to be $-45 \mathrm{~kJ} / \mathrm{mol}$ for adsorption on the LDH surface. For the cationic arginine, with only one carboxylic group and a positive net charge, the energy of adsorption was $-26 \mathrm{~kJ} / \mathrm{mol}$, which is twice higher than that of chloride anion adsorption on the same cationic nanosheet. This fact clearly demonstrates the capability of "soft matter" species to adjust themselves and fit into the surface, minimizing energy of the system. The adsorption of protonated histamine, having no carboxylic groups, on a boehmite nanosheet is also energetically favorable, but the depth of free energy well is quite small at $3.6 \mathrm{~kJ} / \mathrm{mol}$. In the adsorbed state the protonated amino-group of histamine plays the role of proton donor, while the hydroxyl oxygens of the layered hydroxide have the role of proton acceptor, which is unusual. The obtained results represent a small step towards further understanding of the adhesion effects within the hard matter - soft matter contact zone.
\end{abstract}

Key Words: Adhesion, Interface, Soft Matter, Layered Hydroxide, Steered Molecular Dynamics

Received September 15, 2016 / Accepted November 07, 2016

Corresponding author: Alexey A.Tsukanov

Institute of Strength Physics and Materials Science SB RAS, Tomsk, 634055, Russia

E-mail: a.a.tsukanov@yandex.ru 


\section{INTRODUCTION}

In the last decades soft matter engineering has a very rapid development. The importance of the soft matter extends to a wide range of applications in such fields as materials science [1], energetics [2, 3], catalysis [4, 5], and pharmaceutics. It especially stands for biomedicine since biological nano-objects (BNO) such as polypeptides, proteins, bio-membranes as well as almost all biologically-active compounds (drugs, genes, viruses, etc.) are soft-matter systems with flexible, non-uniform and multifunctional surfaces $[6,7]$. One of the main characteristics of the soft matter is its ability to form high-level complicated functional nano-objects from comparatively simple building blocks [8].

On the other hand, certain condensed materials such as naturally occurring layered ceramics (LC), in particular cationic clays, layered double hydroxides (LDH) and metal oxyhydroxides possess special properties such as large surface charge (which allows them to act as host nanoparticles for ionic molecules), large specific surface area, low toxicity, chemical inertness and biocompatibility, which makes them extremely promising in such biomedical/nanomedical applications as drug and gene delivery [9-13]. In addition, lowdimensional aluminum oxyhydroxide nanoparticles are amphoteric compounds with high proton and hydroxyl buffer capacity [14], which can affect the ionic balance in the cellular environment.

The use of LC in the role of hosting "nanocontainers" is explained by the fact that almost all biologically-active molecules (including modified ones) may be intercalated in between hydroxide nanolayers. There are two particularly important aspects in this context: first, interaction effects within the hard matter - soft matter interface (HSI) between the biologically-active compound ("guest") and the layered hydroxide nanosheets ("host") determine the formation of (conditionally) stable organic-inorganic nanohybrids ( $\mathrm{NH})$; second, the interaction between the inorganic outer surface of the nanohybrid and the cell membrane determine the mechanism and efficiency of the cellular uptake of the NH.

Predicting behavior and interaction of hard matter and soft matter subsystems is a challenge. To shed light on several effects which can rise within the hard matter - soft matter interfacial region a series of steered molecular dynamics (SMD) simulations utilizing all-atom models was conducted with different pairs of organic and inorganic nanomaterials.

\section{COMPUTATIONAL TECHNIQUE}

\subsection{Potential energy functional}

To accurately compute any energies and forces of the studied thermodynamic system, suitable potentials need to be selected. In the case of soft matter systems and systems having covalent bonds, the most typical terms in the potential energy functional of the bonded atoms are the bond stretching term, angle bending, Urey-Bradly 1-3 stretching term, dihedral or torsional angle bending and improper rotation or inversions term (Fig. 1). Eq. (1) describes a typical form of potential energy functional with harmonic type of the terms as it implemented in CHARMM [15]: 


$$
\begin{aligned}
& U=\sum_{\text {bonds }} k_{b}\left(b-b_{0}\right)^{2}+\sum_{\text {angles }}\left[k_{\theta}\left(\theta-\theta_{0}\right)^{2}+k_{U B}\left(s-s_{0}\right)^{2}\right]+ \\
& +\sum_{\text {dihedrals }} k_{\varphi}(1+\cos (n \varphi-\delta))^{2}+\sum_{\text {impropers }} k_{\gamma}\left(\gamma-\gamma_{0}\right)^{2}+U_{\text {unbonded }}
\end{aligned}
$$

where the unbounded terms such as Lennard-Jones potential and electrostatic interactions are also included:

$$
U_{\text {unbonded }}=\sum_{i \neq j} \varepsilon_{i j}\left[\left(\frac{\sigma_{i j}}{r_{i j}}\right)^{6}-2\left(\frac{\sigma_{i j}}{r_{i j}}\right)^{12}\right]+\sum_{i \neq j} C \frac{q_{i} \cdot q_{j}}{\varepsilon r_{i j}} .
$$

The most famous all-atom force fields for soft matter systems are GROMACS [16], AMBER [17], DREIDING [18], CHARMM, etc. The potentials for hard matter systems are, e.g., EAM for solid metals [19, 20] and liquid metals [21, 22], CLAYFF for ceramics [23], AIREBO and Tersoff for carbon nanostructures and other compounds [24-26] and others. There is also a hybrid force field INTERFACE, which is suitable for heterogeneous system modeling [27].

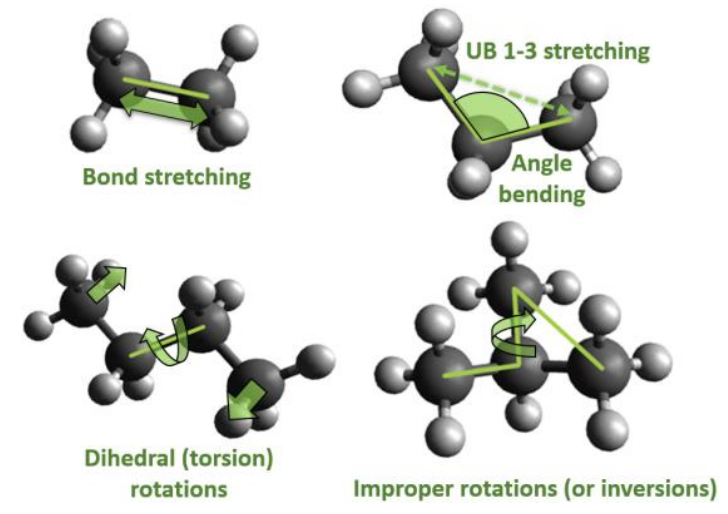

Fig. 1 Typical terms included in the potential energy functional of Force Fields for bonded atoms

For bound atoms, special coefficients are often used reducing the Lennard-Jones and Coulombic terms (so-called 1-2, 1-3, 1-4 special bonds). In addition, to facilitate computations, a cutoff for pairwise interactions with a smoothly decreasing envelopefunction is used. So-called "full electrostatics" (long-range) is usually calculated with the particle-particle particle-mesh (PPPM) [28, 29] or particle-mesh Ewald (PME) methods [30, 31]. In the present study, the cutoff distance of $12 \AA$ for pairwise interaction, with a smooth decrease starting at $10 \AA$ was utilized, as well as the PPPM method for "longrange" Coulombic interactions with a relative accuracy of 0.001 .

Molecular dynamics (MD) modeling of the layered ceramics nanostructures requires not only unbounded terms but also an explicit treatment of the covalent bonds in hydroxyl groups between oxygen and hydrogen atoms [23]. 
To parameterize all molecular systems considered in the present study, the following force fields are used: CHARMM [15] for organic molecules, TIP3P [32] for water molecules, as well as CLAYFF [23] for layered ceramics, with a simple modification according to [33].

\subsection{Free energy of adsorption}

To quantify interactions between different parts of the system or the energy change between two states of the system, free energy analysis is often very useful. In the present study of interaction effects within the HSI region, the combined process free energy estimation (COPFEE) procedure [34] was utilized, which is based on the potential of mean force (PMF) analysis [35] for constant velocity steered MD. The central idea of the COPFEE approach is a combination of two oppositely directed processes, which allows us to reduce the dependence on the velocity of the pulling procedure and to estimate the level of irreversible energy dissipation in comparison with the free energy change. Briefly, to estimate the free energy of adsorption (of some molecule) consider the following combination of two processes: a forward process - forced adsorption, in which an external force is performing the work of translocating an adsorbate from "infinity" (actually some point in the solvent, where the adsorbate is fully hydrated) onto the surface of the adsorbent (Fig. 2); and then a reverse process - forced desorption, when the external force acts to remove the adsorbed molecule/nanoparticle away from the adsorbent surface to any point in the solvent that is equidistant with the initial point (Fig. 2). During both the processes the work done by the external force is integrated, and two free energy profiles (PMF profiles along reaction coordinate) are obtained as functions of $z$, in the direction perpendicular to the adsorbent surface.

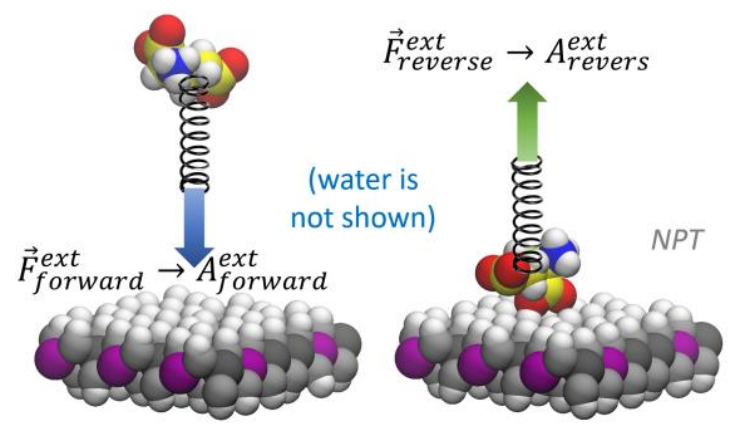

Fig. 2 Two stages of combined process free energy estimation procedure (COPFEE) for an organic anion interacting with an LDH nanosheet: left - forced adsorption of adsorbate on the adsorbent surface under the action of an external force, right forced desorption, the reverse process. During the steered MD simulation, the free end of the abstract spring is moving with constant velocity in the respective direction. Adsorbate colors: yellow $-\mathrm{C}$, white $-\mathrm{H}$, blue $-\mathrm{N}$, red - carboxylic $\mathrm{O}$. Adsorbent colors: purple $-\mathrm{Al}$, black $-\mathrm{Mg}$, gray - hydroxylic $\mathrm{O}$, white $-\mathrm{H}$ 
As is known from thermodynamics, work $A_{\text {ext }}$ of the external force is spent on change of Gibbs free energy $G$ of the system (in case of an isothermal-isobaric ensemble) and on entropy generation (if the process is not reversible):

$$
A_{e x t}=\Delta G+T \delta S .
$$

Writing this for both the forward and reverse processes gives:

$$
\begin{aligned}
& A_{e x t}^{f v d d}=\Delta G_{a d s}+T \delta S^{f v d} \\
& A_{e x t}^{r v s}=-\Delta G_{a d s}+T \delta S^{r v s}
\end{aligned}
$$

where $T$ is known (and constant), both $A^{f w d}$ and $A^{r v s}$ are can be estimated from the SMD simulations, and $\Delta G, \delta S^{\text {fwd }}$ and $\delta S^{r v s}$ are unknown variables. Thus, there are three unknown variables in the system ( $3 b)$ and only 2 equations. To overcome this problem, the following assumption can be made [34]: if the pulling velocities in both forward and reverse processes are equal and sufficiently small, the entropy generation in both processes should also be approximately equal:

$$
\delta S^{f w d} \approx \delta S^{r v s} \text {. }
$$

The system ( $3 \mathrm{~b})$ becomes solvable with this assumption:

$$
\Delta G_{\text {ads }}=\frac{1}{2}\left(A_{\text {ext }}^{\text {fvd }}-A_{e x t}^{r v s}\right)-\frac{T}{2}\left(\delta S^{f v d}-\delta S^{r v s}\right) \approx \frac{1}{2}\left(A_{e x t}^{f v d}-A_{e x t}^{r v s}\right)
$$

If the adsorption is energetically favorable, the external work in forward process $A^{f w d}$ is negative (with a sufficiently small pulling velocity), while $A^{r v s}$ is positive and $\left|A_{\text {ext }}^{r v s}\right| \geq\left|A_{\text {ext }}^{f w d}\right|$. Thus, the free energy of adsorption can be simply estimated as minus the arithmetic mean of the absolute values of the external work for the forward and reverse processes [34]:

$$
\Delta G_{\text {COPFEE }}^{\text {ads }}=-\frac{\left|A_{\text {ext }}^{\text {fwd }}\right|+\left|A_{\text {ext }}^{r v s}\right|}{2} .
$$

\section{RESULTS AND DISCUSSION}

\subsection{Organic anion adsorption on $\mathrm{Mg}_{4} / \mathrm{Al}_{2}-\mathrm{LDH}$ nanosheet}

First we consider a combined adsorption-desorption constant velocity SMD process for the aspartic amino acid anion (in zwitterionic state) on a $\left[\mathrm{Mg}_{4} \mathrm{Al}_{2}(\mathrm{OH})_{12}{ }^{2+}{ }^{\left.2 \mathrm{Cl}^{-}\right]}\right.$ layered double hydroxide nanosheet, which has a strong positive surface charge (about $0.7 \mathrm{C} / \mathrm{m}^{2}$ ) and exposes polar hydroxyl groups on its surface. The aspartic acid anion (ASP) has two carboxylic groups with local negative charges on oxygen atoms. The total charge of the ASP molecule is - $1 \mathrm{e}$. It is convenient for further discourse to put the origin of the coordinate system in the center of mass of the $\mathrm{Mg}_{4} / \mathrm{Al}_{2}-\mathrm{LDH}$ fragment and to orient the z-axis perpendicular to the nanosheet plane.

The PMF profile (the cumulative work as a function of the distance between $\mathrm{Mg}_{4} / \mathrm{Al}_{2}$ LDH nanosheet central plane and ASP center of mass) for the forward process obtained with SMD with a constant pulling velocity $v=0.1 \AA / n s$ is represented by the blue curve in 
Fig. 3. Comparing the reverse process (Fig. 3, green line) it is immediately obvious that the obtained curves are not equal, because of entropy generation during the irreversible part of the process (Fig. 3, 5.6 $<\mathrm{z}<6.4 \AA$ ). It is necessary to note that PMF profiles are relative functions, which is why a zero level must be chosen for both the dependences. Since we are interested in the free energy of adsorption, which is the difference of $G$ between some distant point in the bulk water solution and the point corresponding to the nearest local minimum of PMF, it seems most convenient to choose the zero level at the initial point (in the water) for both the curves.

The too fast perturbation (in comparison with thermal motions) of a single-moleculethick water layer on the adsorbent surface is the most probable reason for the observed entropy generation .This could probably be mitigated with a lower velocity of the pulling process during SMD simulation, while greatly increasing the computational cost of the numerical experiment.

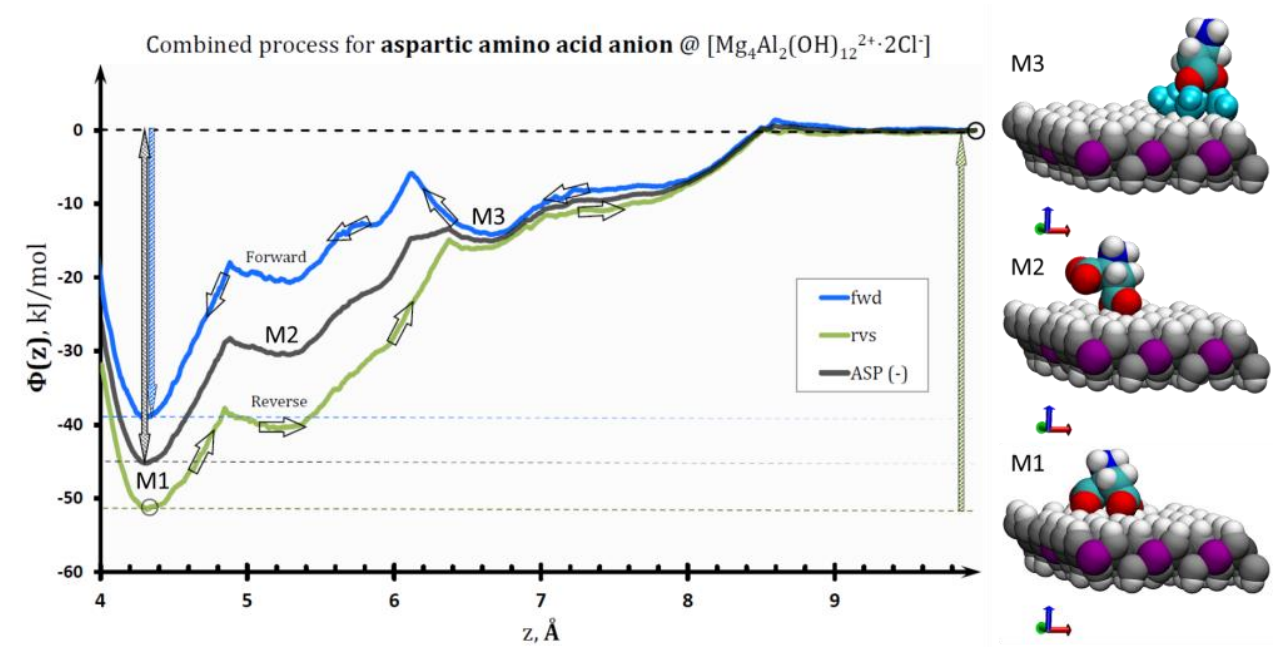

Fig. 3 Free energy of ASP amino acid anion adsorption on LDH using COPFEE method. The profile has three local minima: M3 (6.5-7.0 $\mathrm{A})$ - adsorbate is separated from LDH surface by a single-molecule-thick water layer, M2 (5.0-5.4 $\mathrm{A})$ - first carboxylic group of ASP forms H-bonds with surface OH-groups, M1 (4.2-4.5 - both carboxylic groups of ASP contact with hydroxylic LDH surface completely adsorbed state. Color code is similar to the colors of Fig.2, except that carbon atoms of the adsorbate are in cyan, and water (several molecules in contact zone) in light blue. The rest of the water and other ions are not shown for clarity

Following the procedure described with the assumption Eq. (3c), we obtain an estimate for the free energy of ASP acid anion adsorption on the $\mathrm{Mg}_{4} / \mathrm{Al}_{2}-\mathrm{LDH}$ surface of $-45 \mathrm{~kJ} / \mathrm{mol}$ (minimum M1 in Fig.3). This is a very high value, indicating strong adhesion within the hard matter. Such strong interaction energy within HSI allows the formation of hybrid organic-inorganic multimolecular complexes, as was demonstrated in unbiased (non-steered) molecular dynamics simulations [33]. 
The half-width of the "corridor" between forward and reverse PMF profiles is $\Delta= \pm 6 \mathrm{~kJ} / \mathrm{mol}$, which provides a rough estimate of entropy generation during the forced processes.

\subsection{Arginine and chloride adsorption on cationic nanosheet}

Free energy of adsorption of anionic molecules on an LDH nanosheet was considered in the previous section as well as in other works [33, 34]. The behavior of cationic amino acid residues such as arginine (ARG), lysine and protonated histidine at a cationic nanosheet is also an important question since these also are typical building blocks of proteins and polypeptides, and the possibility of adsorption is not obvious due to electrostatic repulsion. Here the COPFEE procedure was applied to characterize the interaction of arginine amino acid with a $\left[\mathrm{Mg}_{4} \mathrm{Al}_{2}(\mathrm{OH})_{12}{ }^{2+} 2 \mathrm{Cl}^{-}\right]$nanosheet. The all-atom 3D structure of $\mathrm{Mg}_{4} / \mathrm{Al}_{2}-\mathrm{LDH}$ nanosheet was built based on crystallographic data from [36] as in the previous case, wherein the interlayer $\mathrm{CO}_{3}{ }^{2-}$ anion was replaced by dissolved $\mathrm{Cl}^{-}$.

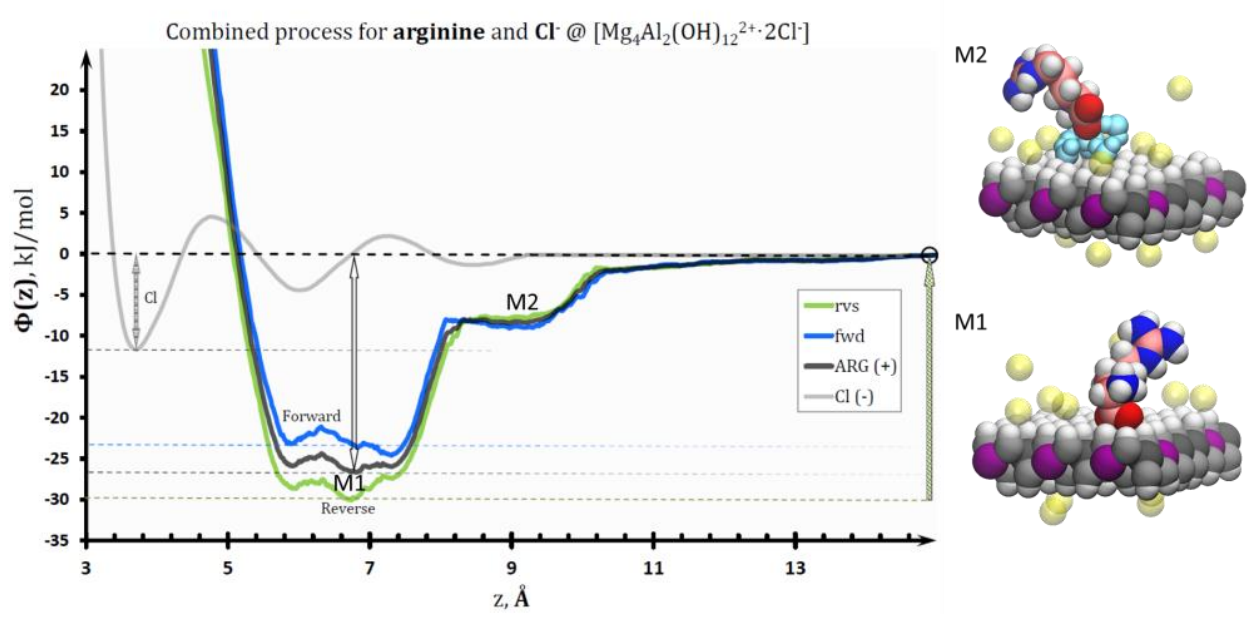

Fig. 4 Amino acid cation (arginine) adsorption on a cationic nanosheet of LDH in comparison with an inorganic anion (chloride ion), using COPFEE method. The gray curve corresponds to COPFEE result for chloride anion adsorption onto the LDH nanosheet. Colors of atoms is similar to the colors of Fig.3, except for carbon atoms of arginine, which are reddish, and chlorine, which is yellow

The obtained profile for ARG adsorption (Fig. 4, black curve) has a minimum M1 (6$7 \AA$ ), in which the carboxylic group of ARG forms several hydrogen bonds with LDH hydroxyl groups, while the positively charged amino group prefers to be located away from the LDH surface (Fig.4). Carboxylic groups of ARG as well as those of ASP amino acid are proton acceptors, while the surface hydroxyl-groups of $\mathrm{LDH}$ are proton donors. There is also a plateau M2 (8-10 $\AA$ ) on the free energy profile, where the arginine carboxylic group is separated from the adsorbent by a one-molecule-thick water layer (Fig. 4, M2 inset). 
Using the COPFEE procedure the surprisingly high estimation for free energy of adsorption of $-26 \mathrm{~kJ} / \mathrm{mol}$ was obtained with the half-width of the forward-reverse PMF corridor $\Delta= \pm 3.5 \mathrm{~kJ} / \mathrm{mol}$. The result means that, despite the fact that ARG is a cation, its adsorption onto the positively charged $\mathrm{Mg}_{4} / \mathrm{Al}_{2}-\mathrm{LDH}$ nanosheet is favorable. This result is quite non-obvious, especially considering that arginine adsorption is even more favorable than adsorption of the chloride anion (Fig.4, grey curve), which has a free energy of -12 $\mathrm{kJ} / \mathrm{mol}$ (using COPFEE as well).

\subsection{Adsorption of carboxyl-less cation on aluminum oxyhydroxide nanosheet}

In both the previous cases, the considered organic ions had one (ARG) or two (ASP) carboxylic groups, which are good terminals (especially in deprotonated state) for $\mathrm{H}$ bonding with the hydroxide surface of LDH. Thus, to understand the behavior of carboxyl-less cationic molecules, a SMD simulation of protonated histamine interacting with an aluminum oxyhydroxide (boehmite) nanosheet was additionally conducted.

The full-atom model of $\mathrm{AlOOH}$ was made using structural data from [37]. Oxyhydroxide model parametrization was performed in accordance with the CLAYFF force field, but null Lennard-Jones parameters were replaced by $r_{0}=0.449 \AA, \varepsilon=0.046$ $\mathrm{kcal} / \mathrm{mol}[15,33]$ to allow proper interactions with the CHARMM subsystem of the model. The net charge of the $\mathrm{AlOOH}$ nanosheet is zero. However, its surface has positive charge due to oriented hydroxylic groups with exposed protons outside.

In the all-atom histamine model, CHARMM-compatible parametrization was utilized, using the SwissParam [38] web-service (http://www.swissparam.ch). Partial atomic charges were obtained using self-consistent field (SCF) calculation in the NWChem package [39] with Hartree-Fock basis $\mathrm{HF} / 6-31 \mathrm{G}^{* *}$ [40, 41].

The histamine molecule in the protonated state has a charge of $+1 \mathrm{e}$, which is concentrated in the amino-group region, where the partial atomic charge of hydrogen atoms in the amino-group is $+0.390 \mathrm{e}$, while the nitrogen contributes $-0.642 \mathrm{e}$.

Using the COPFEE procedure we obtain a value of $-3.6 \mathrm{~kJ} / \mathrm{mol}$ for the free energy of adsorption of protonated histamine (pHST) on the AlOOH nanosheet (Fig. 5, black curve). The depth of the free energy well is comparable to the $k T$ level, which is about $2.5 \mathrm{~kJ} / \mathrm{mol}$ at model temperature $T=310 \mathrm{~K}(p=0.101 \mathrm{MPa}$.). The obtained result shows that, despite electrostatic repulsion, the soft molecule can assume a suitable conformation to allow hydrogen bonding and thus make the adsorbed state energetically favorable, but not as stable as in previous cases. As can be seen in Fig.5, in the adsorbed state pHST is oriented with its $\mathrm{NH}_{3}{ }^{+}$-group towards the boehmite nanosheet. Furthermore, the hydrogen atoms of the boehmite $\mathrm{OH}$-groups that are closest to pHST are pushed apart due to Coulombic repulsion, and one of the $\mathrm{NH}_{3}{ }^{+}$-group protons found contact with hydroxyl oxygens between the hydroxyl hydrogens of the $\mathrm{AlOOH}$.

Using the COPFEE procedure we obtain a value of $-3.6 \mathrm{~kJ} / \mathrm{mol}$ for the free energy of adsorption of protonated histamine (pHST) on the AlOOH nanosheet (Fig. 5, black curve). The depth of the free energy well is comparable to the $k T$ level, which is about $2.5 \mathrm{~kJ} / \mathrm{mol}$ at model temperature $T=310 \mathrm{~K}(p=0.101 \mathrm{MPa}$.). The obtained result shows that, despite electrostatic repulsion, the soft molecule can assume a suitable conformation to allow hydrogen bonding and thus make the adsorbed state energetically favorable, but not as stable as in previous cases. As can be seen in Fig.5, in the adsorbed state pHST is 
oriented with its $\mathrm{NH}_{3}{ }^{+}$-group towards the boehmite nanosheet. Furthermore, the hydrogen atoms of the boehmite $\mathrm{OH}$-groups that are closest to pHST are pushed apart due to Coulombic repulsion, and one of the $\mathrm{NH}_{3}{ }^{+}$-group protons found contact with hydroxyl oxygens between the hydroxyl hydrogens of the $\mathrm{AlOOH}$.

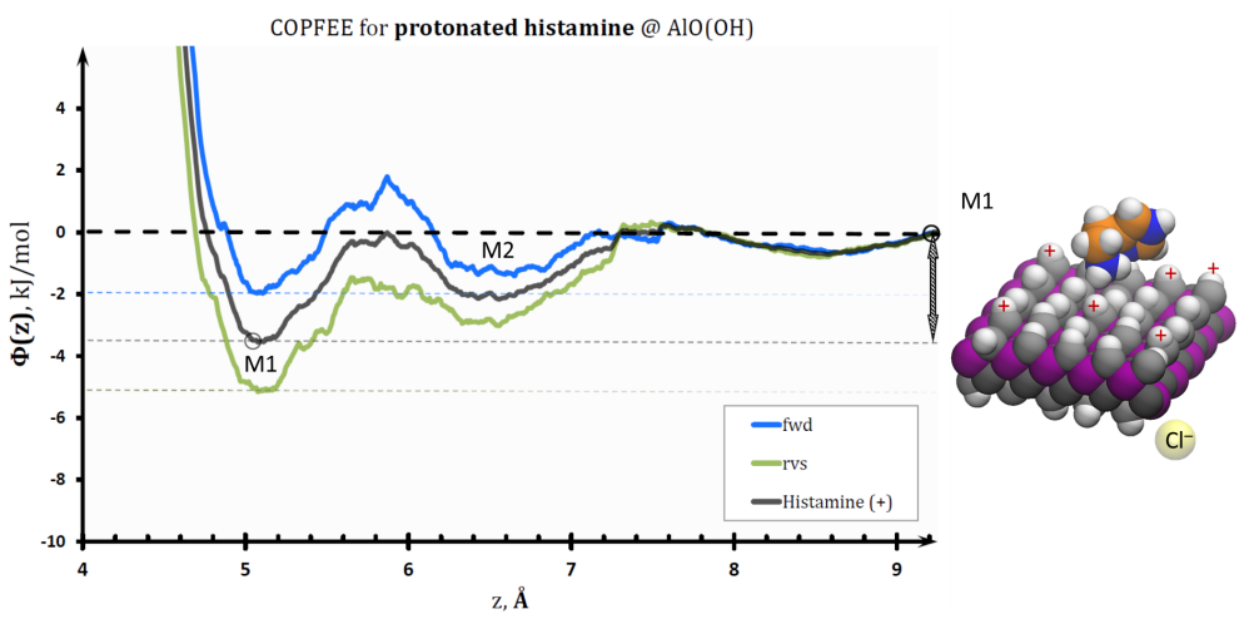

Fig. 5 Combined process for histamine (in protonated state) adsorption-desorption on an aluminum oxyhydroxide nanosheet. Colors for atoms: aluminum - purple, bridging oxygen - black, hydroxyl oxygen - grey, hydrogen - white, nitrogen - blue, carbon - orange, chlorine - yellow. Water is not shown

Thus, the current case is unusual in that the adsorbed molecule is a proton donor, while the hydroxyl oxygens of the nanosheet are proton acceptors, unlike the previous cases.

\section{CONCLUSION}

In the present work, three different soft matter - hard matter couples of nanomaterials are studied to reveal regularities in the nanohybrid formation and the stability of supermolecular complexes, as well as to analyze the energy aspects of adhesion between bio-molecules and layered metal hydroxides, which holds great promise in a wide range of biomedical applications.

The conducted SMD study shows that anionic and cationic bio-molecules can form (conditionally) stable nanocomplexes with positively charged layered hydroxide nanosheets, even despite the electrostatic repulsion in the case of a cationic adsorbate. Since both anionic and cationic amino acids (in zwitterionic state) are capable of being adsorbed on the $\left[\mathrm{Mg}_{4} \mathrm{Al}_{2}(\mathrm{OH})_{12}{ }^{2+}{ }^{2 \mathrm{Cl}^{-}}\right]$surface, all 20 amino acids in zwitterionic state could exhibit the same behavior. Comparing the free energy of adsorption of ASP, having two carboxylic groups, and ARG with one carboxylic group onto $\left[\mathrm{Mg}_{4} \mathrm{Al}_{2}(\mathrm{OH})_{12}{ }^{2+}{ }^{\left.2 \mathrm{Cl}^{-}\right]}\right.$ surface, it may be concluded that each deprotonated carboxylic group adds about 20$25 \mathrm{~kJ} / \mathrm{mol}$ to the depth of the free energy well; however, the exact mechanism can be very 
complicated, and many different aspects such as charge, shape, size, hydrophilicity and so on must be taken into consideration.

Comparing the free energy profiles obtained for ARG and chlorine adsorption, it seems that the flexibility of the molecule and non-uniform spatial distribution of the electric charge on the molecule allow it to "sneak" in between the hydroxylic surface and the surrounding water molecules, thus minimizing the energy of the system more efficiently than the simple chlorine anion.

Looking at the interaction of pHST with the boehmite nanosheet, it can be concluded that layered metal hydroxides can act not only as proton donors in hydrogen bonding but also as proton acceptors. In this case, the depth of free energy well is much lower, however. Moreover, in the case of a deprotonated surface hydroxyl group, the remaining oxygen atom can be a stronger proton acceptor, which may produce strong adsorption sites in defective zones, edges and cleavages.

As shown in the considered cases, the interactions within the interface of hard and soft matter may be quite nontrivial, while playing a crucial role in the formation of hybrid multimolecular nanocomplexes and in the modification of cellular environments via selective adsorption of bio-molecules and ions, both of which is important in modern nanomedical and biomedical applications.

Despite first steps in this direction, the interface between living and nonliving matter remains a rich object for multidisciplinary investigation, including contact mechanics, chemistry, biology, medicine and computational methods, especially molecular simulations.

All MD simulations are performed using the LAMMPS package (Sandia National Laboratory, USA) [42] on the Lomonosov-1 cluster Supercomputing Center of Lomonosov Moscow State University (MSU, Russia) [43]. VMD [44] and Avogadro [45] packages are used in systems preparation and visualization.

Acknowledgements: The paper is a part of the research done within the Russian Science Foundation Grant No. 14-23-00096. The work was supported by the Fundamental Research Program of the state academies of sciences on 2013-2020 years. The authors would like to thank Mikhail Popov (Berlin University of Technology, Germany) for useful ideas, discussions and help with the preparation of the paper.

\section{REFERENCES}

1. Li, C., Strachan, A., 2011, Molecular dynamics predictions of thermal and mechanical properties of thermoset polymer EPON862/DETDA, Polymer, 52(13), pp. 2920-2928.

2. Shamardina, O., Kulikovsky, A.A., Chertovich, A.V., Khokhlov, A.R., 2012, A Model for HighTemperature PEM Fuel Cell: The Role of Transport in the Cathode Catalyst Layer, Fuel Cells, 12(4), pp. 577-582.

3. Komarov, P.V., Khalatur, P.G., Khokhlov, A.R., 2013, Large-scale atomistic and quantum-mechanical simulations of a Nafion membrane: Morphology, proton solvation and charge transport, Beilstein journal of nanotechnology, 4(1), pp. 567-587.

4. Chughtai, A.H., Ahmad, N., Younus, H.A., Laypkov, A., Verpoort, F., 2015, Metal-organic frameworks: versatile heterogeneous catalysts for efficient catalytic organic transformations, Chemical Society Reviews, 44(19), pp. 6804-6849.

5. de Clippel, F., Dusselier, M., Van de Vyver, S., Peng, L., Jacobs, P.A., Sels, B.F., 2013, Tailoring nanohybrids and nanocomposites for catalytic applications, Green Chemistry, 15(6), pp. 1398-1430. 
6. Zeng, X., Li, S., 2012, A three dimensional soft matter cell model for mechanotransduction, Soft Matter, 8(21), pp. 5765-5776.

7. Poon, W.C., Andelman, D. (eds.)., 2006, Soft condensed matter physics in molecular and cell biology, CRC Press.

8. Cranford, S.W., Buehler, M.J., 2012, Biomateriomics, Vol. 165, Springer Science Business Media.

9. Li, L., Gu, W., Chen, J., Chen, W., Xu, Z.P., 2014, Co-delivery of siRNAs and anti-cancer drugs using layered double hydroxide nanoparticles, Biomaterials, 35(10), pp. 3331-3339.

10. Jain, S., Datta, M., 2014, Montmorillonite-PLGA nanocomposites as an oral extended drug delivery vehicle for venlafaxine hydrochloride, Applied Clay Science, 99, pp. 42-47.

11. Hu, H., Xiu, K.M., Xu, S.L., Yang, W.T., Xu, F.J., 2013, Functionalized layered double hydroxide nanoparticles conjugated with disulfide-linked polycation brushes for advanced gene delivery, Bioconjugate chemistry, 24(6), pp. 968-978.

12. Li, D., Zhang, Y.T., Yu, M., Guo, J., Chaudhary, D., Wang, C.C., 2013, Cancer therapy and fluorescence imaging using the active release of doxorubicin from MSPS/Ni-LDH folate targeting nanoparticles, Biomaterials, 34(32), pp. 7913-7922.

13. Jakubikova, B., Kovanda, F., 2010, Utilization of layered double hydroxides in medical applications, Chem. List, 104, pp. 906-912.

14. Lozhkomoev, A.S., Kazantsev, S.O., Lerner, M.I., Psakhie, S.G., 2016, Acid-base and adsorption properties of the AlOOH $2 \mathrm{D}$ nanostructures as factors for regulating parameters of model biological solutions, Nanotechnologies in Russia, 11(7-8), pp. 506-511.

15. MacKerell, A.D., Jr., et al, 1998, All-atom Empirical Potential for Molecular Modeling and Dynamics Studies of Proteins. J. Phys. Chem. B, 102, pp. 3586-3616.

16. Berendsen, H.J.C., van der Spoel, D., van Drunen, R., 1995, GROMACS: A Message-Passing Parallel Molecular Dynamics Implementation, Comp. Phys. Comm., 91, pp. 43-56.

17. Pearlman, D.A., et al, 1995, AMBER, a Package of Computer Programs for Applying Molecular Mechanics, Normal Mode Analysis, Molecular Dynamics and Free Energy Calculations to Simulate the Structural and Energetic Properties of Molecules, Comp. Phys. Comm., 91, pp. 1-41.

18. Mayo, S.L., Olafson, B.D., Goddard, W.A., 1990, DREIDING: A Generic Force Field for Molecular Simulations, J. Phys. Chem., 94, pp. 8897-8909.

19. Daw, M.S., Baskes, M.I., 1986, Semiemperical, Quantum Mechanical Calculation of Hydrogen Embrittlement in Metals, Phys. Rev. Lett., 50, pp. 1285-1288.

20. Foiles, S.M., Baskes, M.I., Daw, M.S., 1986, Embedded-atom-method functions for the fcc metals $\mathrm{Cu}$, Ag, Au, Ni, Pd, Pt, and their alloys, Phys. Rev. B, 33, pp. 7983-7991.

21. Mendelev, M.I., Han, S., Srolovitz, D.J., Ackland, G.J., Sun, D.Y., Asta, M., 2003, Development of new interatomic potentials appropriate for crystalline and liquid iron, Phil. Mag., 83, pp. 3977-3994.

22. Belashchenko, D.K., 2006, Application of the Embedded Atom Model to Liquid Metals: Liquid Mercury, High Temperature, 44, pp. 675-686.

23. Cygan, R.T., Liang, J.-J., Kalinichev, A.G., 2004, Molecular Models of Hydroxide, Oxyhydroxide, and Clay Phases and the Development of a General Force Field, J. Phys. Chem. B, 108, pp. 1255-1266.

24. Stuart S.J., Tutein A.B., Harrison J.A., 2000, A reactive potential for hydrocarbons with intermolecular interactions, J. Chem. Phys., 112, 14, pp. 6472.

25. Tersoff J., 1988, Empirical Interatomic Potential for Carbon, With Application to Amorphous Carbon, Phys. Rev. Lett., 61, pp. 2879-2882.

26. Prodanov N.V., Khomenko A.V., 2010, Computational investigation of the temperature influence on the cleavage of a graphite surface, Surface Science, 604, 7-8, pp. 730-740.

27. Heinz, H., Lin, T.J., Kishore Mishra, R., Emami, F.S., 2013, Thermodynamically consistent force fields for the assembly of inorganic, organic, and biological nanostructures: the INTERFACE force field, Langmuir, 29(6), pp. 1754-1765.

28. Hockney, R.W., Goel, S.P., Eastwood, J.W., 1973, A 10000 particle molecular dynamics model with long range forces, Chemical Physics Letters, 21(3), pp. 589-591.

29. Hockney, R.W., Eastwood, J.W., 1988, Computer simulation using particles, CRC Press.

30. Ewald, P.P., 1921, Ewald summation, Ann. Phys, 369, pp. 253.

31. de Leeuw, S.W., Perram, J.W., Smith, E.R., 1980, Simulation of electrostatic systems in periodic boundary conditions. I. Lattice sums and dielectric constants, In Proceedings of the Royal Society of London A: Mathematical, Physical and Engineering Sciences, The Royal Society, 373, 1752, pp. 27-56.

32. Jorgensen, W.L., Chandrasekhar, J., Madura, J.D., Impey, R.W., Klein, M.L., 1983, Comparison of simple potential functions for simulating liquid water, J. Chem. Phys., 79(2), pp. 926-935. 
33. Tsukanov, A.A., Psakhie, S.G., 2016, Energy and structure of bonds in the interaction of organic anions with layered double hydroxide nanosheets: A molecular dynamics study, Scientific reports, 6, pp. 19986

34. Tsukanov, A.A., Psakhie, S.G., 2016, Adsorption of charged protein residues on an inorganic nanosheet: Computer simulation of LDH interaction with ion channel, In Physics of Cancer: Interdisciplinary Problems and Clinical Applications (PC'16), AIP Publishing, 1760, 1, pp. 020066

35. Izrailev, S., Stepaniants, S., Isralewitz, B., Kosztin, D., Lu, H., Molnar, F., ..., Schulten, K., 1999, Steered molecular dynamics, In Computational molecular dynamics: challenges, methods, ideas. Springer Berlin Heidelberg, pp. 39-65.

36. Arakcheeva, A.V., Pushcharovskii, D.Yu., Atencio, D., Lubman, G.U., 1996, Crystal structure and comparative crystal chemistry of Al2 $\mathrm{Mg} 4(\mathrm{OH}) 12(\mathrm{CO} 3) 3 \mathrm{H} 2 \mathrm{O}$, a new mineral from the hydrotalcitemanasseite group, Crystallography Reports, 41, pp. 972-981.

37. Noel, Y., Demichelis, R., Pascale, F., Ugliengo, P., Orlando, R., Dovesi, R., 2009, Ab initio quantum mechanical study of $\gamma$-AlOOH boehmite: structure and vibrational spectrum, Physics and Chemistry of Minerals, 36(1), pp. 47-59.

38. Zoete, V., Cuendet, M.A., Grosdidier, A. Michielin, O., 2011, SwissParam, a fast force field generation tool for small organic molecules, J. Comput. Chem. 32, pp. 2359-2368.

39. Valiev, M., Bylaska, E.J., Govind, N., Kowalski, K., Straatsma, T.P., Van Dam, H.J., ... De Jong, W.A., 2010, NWChem: a comprehensive and scalable open-source solution for large scale molecular simulations, Computer Physics Communications, 181(9), pp. 1477-1489.

40. Krishnan, R., Binkley, J.S., Seeger, R. Pople, J.A., 1980, Self-consistent molecular orbital methods. XX. A basis set for correlated wave functions, J. Chem. Phys. 72, pp. 650-655.

41. Hariharan P.C., Pople, J.A., 1973, Influence of Polarization Functions on MO Hydrogenation Energies, Theor. Chim. Acta, 28, pp. 213-222.

42. Plimpton, S., 1995, Fast parallel algorithms for short-range molecular dynamics, J. Comp. Phys., 117, pp. $1-19$.

43. Sadovnichy, V., Tikhonravov, A., Voevodin, Vl. Opanasenko, V., 2013, “Lomonosov”: Supercomputing at Moscow State University, In Contemporary High Performance Computing: From Petascale toward Exascale (Chapman Hall/CRC Computational Science), Boca Raton, USA, CRC Press, pp. 283-307.

44. Humphrey, W., Dalke, A. Schulten, K., 1996, VMD - visual molecular dynamics, J. Molec. Graphics 14 , pp. 33-38.

45. Hanwell, M.D., Curtis, D.E., Lonie, D.C., Vandermeersch, T., Zurek, E., Hutchison, G.R., 2012, Avogadro: an advanced semantic chemical editor, visualization, and analysis platform, Journal of cheminformatics, 4(1), pp. 1. 